\title{
Attention shifting between global and local target levels: The persistence of level-repetition effects
}

\author{
Ronald Hübner \\ Technische Universität Braunschweig, Germany
}

\begin{abstract}
The aim of the reported experiments was to investigate the persistence of global/ local level-repetition effects in an identification task with hierarchical stimuli. In the first experiment the trial rate was self paced, and cues were available during the unconstrained preparation interval. Nevertheless, significant level-repetition effects occurred, which were similar for shifts from global to local, and vice versa. Also, the role of the spatial-frequency content of the stimuli was examined. but no indication was found that it affected the repetition effects. In the second experiment, the time and the cue information available for preparation as well as the interval between cue and stimulus (ISI) was varied. It turned out that the response times decreased with an increasing ISI. However, the level-repetition effects were unaffected. In all, the level-repetition effects turned out to be largely independent of voluntary control mechanisms.
\end{abstract}

\section{INTRODUCTION}

Performance often requires the selection of certain stimuli or at least of some of their attributes, and an important question is how this selection is accomplished. Many studies revealed that the efficiency of stimulus selection can be increased by allocating or shifting attention to the input streams or channels that encode the relevant stimulus information, and that the allocation is affected by bottom-up (or stimulus-driven) as well as by top-down (or goal-directed) processes (for overviews see e.g. Egeth \& Yantis, 1997; Johnston \& Dark, 1986; Kinchla, 1992; Shiffrin, 1988). In a similar manner, Posner (e.g. 1980) has

Correspondence should be sent to R. Hübner, Universität Konstanz, Fachgruppe Psychologie, Fach 29, D-78457 Konstanz, Germany. Email: ronald.huebner@uni-konstanz.de

I am very grateful to Dirk Vorberg and Gerriet Backer for their valuable suggestions and comments on earlier versions of this paper. The research reported in this article was supported by a grant (Hu 432/5-1) from the Deutsche Forschungsgemeinschaft (DFG). 
distinguished between exogenous and endogenous control modes of attention shifting, respectively.

These concepts are rather general and can be applied to spatial attention, where they mainly were developed, as well as to other areas such as, for instance, attention to global and local levels of hierarchical stimuli (e.g. Hübner, 1997; Lamb \& Yund, 1996a; Ward, 1982), which is the topic of the present paper. The question addressed here is: What determines the efficiency of level selection? Does it suffice to assume exogenous and endogenous attentional control mechanisms, or are there some other relevant factors? One phenomenon that might be informative in this respect are level-repetition effects, i.e. the fact that performance is usually increased if the target level on a given trial is the same as that in the preceding trial (e.g. Hübner, 1997; Lamb, London, Pond, \& Whitt, 1998; Lamb \& Yund, 1996b; Robertson, 1996; Ward, 1982). These effects were addressed by the present experiments. Specifically, I investigated whether the level repetition effects can be overcome by attentional control. Before the experiments are described in detail, however, the global/ local paradigm shall be introduced.

Most global/local studies have used compound letters as stimuli. They consist of a global letter form composed from identical smaller local letters (see Figure 1). Usually, the task of the participants is to identify either the letter at the global or that at the local level. If the letter at the non-target level is response compatible or response incompatible, then the stimulus is considered as consistent or inconsistent, respectively. The majority of experiments have revealed that, under various conditions, the global letters are identified faster than the local ones, and there is often an interference asymmetry in the sense that the global information interferes with local processing, but not vice versa. This pattern of results, i.e. the global response-time advantage and the interference asymmetry, led Navon (1977), to propose that the information at both levels is processed sequentially, where the global features are processed first. Further, he assumed that sensory mechanisms are responsible for both effects. For instance, it has been proposed that the spatial-frequency characteristic of our visual system is a possible candidate for such a mechanism (see Hughes, Nozawa, \& Kitterle, 1996 for an overview).

However, the sensory hypothesis was questioned, and alternatively, attentional accounts have been proposed (e.g. Hoffman, 1980; Kinchla, SolisMacias, \& Hoffman, 1983; Miller, 1981; Ward, 1982). Particularly levelrepetition effects, observed first by Ward (1982), were thought to support an attentional explanation of the global advantage. He employed a doubleidentification task, where the participants had to respond to two hierarchical stimuli in short succession. When the target level remained the same for the first and second judgement, then the responses were considerably faster relative to the condition in which attention had to be shifted between the levels, an effect that he called "level-readiness effect". Local-level repetition could even 


\section{$\mathrm{HHHHHH}$}

H

$\mathrm{H}$

HHHHHHH

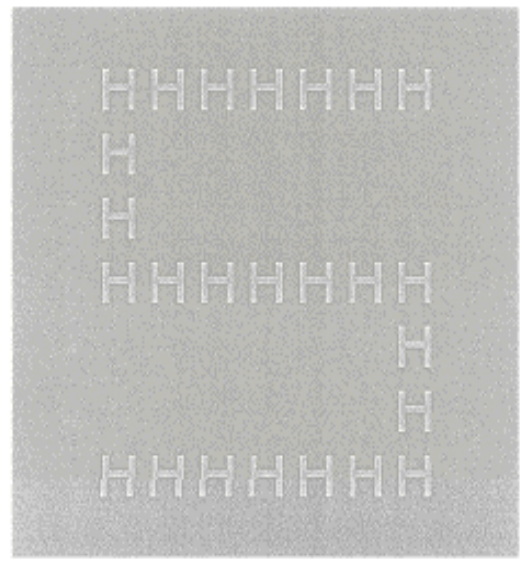

FIG. 1. An example of the hierarchical letters used in the experiments. The stimulus in the lower part of the figure is a highpass-filtered version of the stimulus in the upper part.

lead to a local response-time advantage, which led Ward (1982) to assume that not only the saliency of the features at a given level affect the selection efficiency, but also the attentional resources allocated to that level.

Subsequently, several studies have reported level-repetition effects. For instance, Robertson, Egly, Lamb, and Kerth (1993) and Robertson (1996) found sequential effects for inter-trial intervals (ITIs) of up to $3 \mathrm{sec}$. As in Ward (1982), they observed that the effects were similar for shifts from local to global or vice versa. Especially from this symmetry Robertson et al. (1993) concluded that level-repetition effects are due to categorical priming of the levels, i.e. they 
assumed that level priming does not affect a regional mechanism, such as the size of the attentional window. Rather, priming was supposed to occur at an abstract stage where the levels are represented as categories. In a later paper, Robertson (1996) elaborated this hypothesis by assuming that the priming is due to a certain weighting of low and high spatial-frequency channels. She tested the hypothesis by applying stimuli that did not possess low spatial frequencies, and, indeed, when these stimuli alternated with normal stimuli, then no sequential effects occurred. However, as she admitted, not only the spatial-frequency content varied from trial to trail in this situation, but also other attributes such as contrast and brightness. Lamb and Yund (1996a) conducted a better controlled experiment, and could not support the hypothesis that the spatial-frequency content of the stimuli is relevant for level-repetition effects. In a recent paper, Lamb, Yund, and Pond (1999) provided some evidence that learning might play a role in this respect.

Instead of assuming that the weighting of spatial-frequency channels is responsible for the level-repetition effect, Lamb and Yund (1996b) proposed, more generally, that the effect is due to the activation of level-specific neural mechanisms. According to this "mechanism activation" hypothesis, the repetition benefits result from the fact that, when the information at a certain level has been processed before, the appropriate mechanism is already active and the processing of the current stimulus benefits from this activation. Voluntary control mechanisms are thought to have no effect, or at least to be unable to overcome the automatic activation. By comparing three conditions Lamb et al. (1998) tested this hypothesis. In one condition the target level was constant within a block of trials, and in another it was randomized across trials. Finally, in a third condition the target levels were alternating. Lamb et al. (1998) employed two target letters and two distractor letters, where each stimulus contained a target letter at one level, and a distractor letter on the other. Since there was no cue in the randomized-level condition, the participants had to distribute their attention to the two levels, or to focus it on one, and, if it was the wrong level, to shift to the other.

Although the target level was not predictable in the randomized condition, it was predictable in the other two conditions. Thus, if the participants could allocate their attention in advance, then the randomized condition should produce the worst performance. However, predictability had no effect. On the other hand, the percentage of level repetitions had a severe effect. The best performance occurred in the blocked condition (100\% repetitions), and the worst in the alternating condition ( $0 \%$ repetitions). The performance in the randomized condition (50\% repetitions) was in between. Thus, the more level repetitions, the faster the responses. This suggests that voluntary control had no effect, or, at least, it could not overcome the automatic process. 
This result is surprising, since there are other results demonstrating that the validity of cues can affect performance (e.g. Robertson et al., 1993), which suggests that voluntary attention shifts are possible. One problem with the Lamb et al.'s (1998) procedure is that focused attention was not required. Since only one target was present, the participants could perform the task even if they did not attend to a certain level in advance (and whether participants focused or distributed their attention was not under the control of the experimenter). On the other hand, it could be that appropriate visual cues are necessary, which can help to focus attention to the expected level.

Given these questions and problems, level-repetition effects were investigated again here with a procedure where potential targets occurred at both levels so that the participants had to process the cue and to attend to the indicated level in order to perform the task correctly. Cues were rectangles of a size corresponding to that of the respective target pattern. It was assumed that these cues allowed subjects to adjust the focus of attention to the specified size in advance. Additionally, to favour voluntary control mechanisms even more, a self-paced procedure was employed, i.e. the cue was available at the beginning of each trial, and the participants could decide by themselves when to receive the stimulus. The duration of this interval, which was interpreted as preparation time, was registered and analysed as well.

To test whether level repetition has still an effect under these conditions, a condition with a constant target level and one with a randomized target level were used. Additionally, to see whether a deterministic level order differs from a random one, a condition with an alternating level of run length 2 was added. If only the percentage of level repetitions determines the size of the repetition effect, then the randomized condition should produce the same effect size as the "runs" condition.

Additionally, the first experiment served for testing the hypothesis that the spatial-frequency content of the stimuli affects the level-repetition effects (cf. Robertson, 1996). Although most of the studies have used contrast-balanced stimuli for testing this hypothesis, I employed highpass-filtered stimuli here. Using contrast-balanced stimuli might be problematic. On the one hand, there are different methods of constructing these patterns. For instance, Lamb and Yund (1996a) used white lines on a grey background, and simply added black lines, so that the average luminance was equal to that of the background. Different from this method, Robertson (1996) used contrast-balanced dots. On the other hand, contrast balancing not only reduces low spatial frequencies, but introduces additional edges and, therefore, additional energy at high spatial frequencies (see the amplitude spectrum in Lamb \& Yund, 1996a). Thus, additional to the three different level-repetition conditions, the spatial-frequency content of the stimuli was varied. 


\section{EXPERIMENT 1}

\section{Method}

Participants. Eight persons (four male and four female), who ranged in age between 19 and 28 years, participated in the experiment. All had normal or corrected-to-normal visual acuity.

Apparatus. The stimuli were presented on a 19" colour monitor (Miro, Type GDM-1965) with a resolution of $1280 \times 1024$ pixels, which was connected to a graphics-board (Miro-Tiger) with 256 grey levels and a refresh rate of $75 \mathrm{~Hz}$ (non-interlaced). A personal computer (PC) served for controlling stimulus presentation and response registration.

Stimuli. Four different letters, "H", "U", "A", and "S", were used, and mapped to two response categories ("H", "S") and ("A", "U"). By combining all letters, 16 compound stimuli were created, where global letters were constructed from identical local letters in a $7 \times 7$ grid. A stimulus was consistent if the letters at both levels belonged to the same response category; otherwise it was inconsistent.

At a viewing distance of $127 \mathrm{~cm}$ the global letters extended a visual angle of $1.96^{\circ}$ horizontally and of $2.35^{\circ}$ vertically, and the local letters extended $0.21 \times 0.24^{\circ}$, respectively. The width of the lines composing the local letters was $0.05^{\circ}$. An example stimulus is given in Figure 1. The luminance of the letters was $82 \mathrm{~cd} / \mathrm{m}^{2}$, and the contrast (Michelson contrast) was $52 \%$. Background luminance was set to $41 \mathrm{~cd} / \mathrm{m}^{2}$.

Additionally there were highpass-filtered versions of the stimuli. The unfiltered and highpass-filtered versions of an example stimulus are given in Figure 1. Highpass-filtering was accomplished by weighting the spectrum of the filled letters with that of the highpass version of a Gaussian filter (i.e. 1 minus the response function of a standard lowpass Gaussian filter) with a standard deviation of $8.20 \mathrm{cpd}$, and by transforming the result back to the spatial domain. The contrast (Michelson contrast) of the filtered letters was $99 \%$.

Procedure. The task of the participants was to classify the letters according to the response categories by pressing one out of two response buttons with their index or middle finger, respectively, of the same hand. Mapping of the categories to the response keys (or fingers) were counterbalanced across subjects. Each trial started with the appearance of a fixation-cross and a cue, which were both centred on the screen. The cue consisted of the outline of a rectangle, in a size corresponding to that of the respective target level (letter). After the participants had started the trial by pressing a start key with the hand that was not used for responding to the target stimuli, a blank screed appeared for $400 \mathrm{msec}$, followed by the stimulus, which was randomly exposed $0.95^{\circ}$ (measured to the 
centre of the stimulus) left or right of the fixation point for $187 \mathrm{msec}$. The position was lateralized and randomized to obtain a large global response-time advantage (cf. Lamb \& Robertson, 1988). 1000msec after the response the cue for the next trial appeared and remained on the screen until the participants pressed the start key again. The duration of this interval was registered and interpreted as preparation time.

There were three blocked level-repetition conditions: The target level was either constant within a block of trials, randomized, or alternating in runs of length two. Thus, the factors were stimulus type (unfiltered, highpass filtered), target level (global, local), consistency (consistent, inconsistent), and levelrepetition (constant, randomized, runs). The stimulus-type factor was also blocked.

There were two blocks of 128 trials for the runs and the randomized conditions (one block normal, one highpass), and four blocks of 64 trials for the last main condition (global, local times normal, highpass). All blocks were presented five times, where their order was randomized for each participant. The first block of each condition was considered as training, and the corresponding data were discarded. Altogether, 128 trials for each individual condition and participant were included in the analysis.

\section{Results}

Response times. The mean latencies of the correct responses were subjected to a four-factor ANOVA with repeated measures on all factors: level repetition (constant, randomized, runs), stimulus type (unfiltered, filtered), target level (global, local), and consistency (consistent, inconsistent).

Level repetition had a significant effect, $F(2,14)=15.7, p<.001, M S E=$ 903. The responses in the blocked-level condition were faster $(525 \mathrm{msec})$ than those in the other two conditions (551 msec in each condition). Also, there was a significant effect of stimulus type, $F(1,7)=80.2, p<.001, M S E=5691$; responses were considerably faster to unfiltered stimuli $(494 \mathrm{msec})$ than to highpass-filtered stimuli $(591 \mathrm{msec})$. Further, the target-level factor [global: $512 \mathrm{msec}$, local: $572 \mathrm{msec} ; F(1,7)=30.7, p<.001, M S E=5605]$, and the consistency factor [consistent: $534 \mathrm{msec}$, inconsistent: $551 \mathrm{msec} ; F(1,7)=47.1$, $p<.001, M S E=307]$ were significant.

The two-way interactions of stimulus type $\times$ consistency [unfiltered: $482 \mathrm{msec}$ versus $505 \mathrm{msec}$, highpass-filtered: $585 \mathrm{msec}$ versus $597 \mathrm{msec} ; F(1$, $7)=21.8, p<.001, M S E=73.8$ ], and target level $\times$ consistency [global: $511 \mathrm{msec}$ versus $514 \mathrm{msec}$, local: $557 \mathrm{msec}$ versus $588 \mathrm{msec} ; F(1,7)=11.6, p<$ $.05, M S E=797]$ were significant. However, the highpass-filtered stimuli did not reduce the latency difference between responses to global and local targets significantly, relative to the unfiltered stimuli [unfiltered: $68 \mathrm{msec}$, filtered: $52 \mathrm{msec} ; F(1,7)=1.27, p<.29$ ]. 


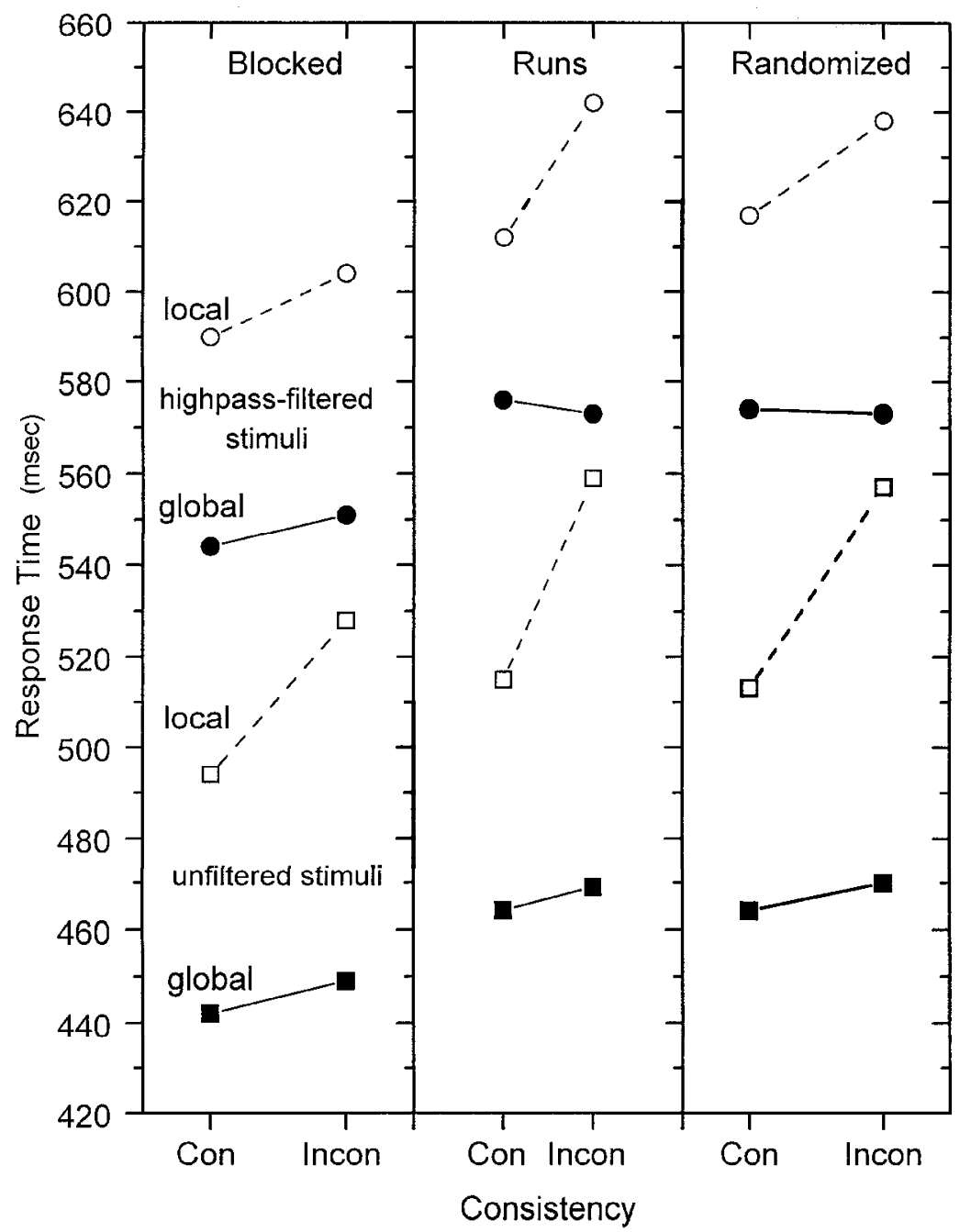

FIG. 2. Mean response-time data for the different conditions and stimuli in Experiment 1.

Of the three-way interactions, that between stimulus type, target level, and consistency was significant, $F(1,7)=17.9, p<.01, M S E=31.5$. The interference asymmetry between the global and the local level was smaller for filtered stimuli than for unfiltered stimuli, as can be seen by considering Figure 2. A post hoc test revealed that the interference effect for the local level of the filtered stimuli was still significant, $F(1,7)=15.2, p<.01, M S E=35.1$.

Finally, also the three-way interaction between level repetition, target level, and consistency was significant, $F(2,14)=4.10, p<.05, M S E=84.6$. For analysing this interaction, the individual level-repetition conditions were analysed 
separately. Although there was a significant interaction between target level and consistency for the alternate-runs condition [global: $520 \mathrm{msec}$ versus $521 \mathrm{msec}$, local: $564 \mathrm{msec}$ versus $600 \mathrm{msec} ; F(1,7)=16.2, p<.01, M S E=$ 1258], and for randomized target levels [global: $519 \mathrm{msec}$ versus $522 \mathrm{msec}$, local: $565 \mathrm{msec}$ versus $597 \mathrm{msec} ; F(1,7)=8.51, p<.05, M S E=846]$, the interaction failed to reach significance for the blocked condition (see Figure 2).

Preparation times. Preparation times were subjected to an ANOVA with level repetition and target level as factors. The first factor was significant, $F(2$, $14)=10.3, p<.01, M S E=37,787$. A constant target led to the shorter preparation times $(330 \mathrm{msec})$ than the alternate-runs $(395 \mathrm{msec})$. Bonferroni $t$-tests for paired comparisons computed across the different conditions and participants revealed that this difference was significant, $t(63)=5.55, p<.001$. Randomizing the target level produced the longest preparation times $(485 \mathrm{msec})$, which were significantly longer than those in the alternate-runs condition, $t(63)=$ $7,93, p<.001$.

Also the target-level factor was significant, $F(1,7)=8.98, p<.05, M S E=$ 8709. Preparation for responding to the global target level took less time $(383 \mathrm{msec})$ than preparation for responding to the local target level $(423 \mathrm{msec})$.

The preparation data are depicted in Figure 3. Since also other results are shown in this figure, the data were classified by whether the target level was the same as that in the previous trial (no-shift trials) or different (shift trials).

Alternate-runs condition. The response-time and preparation-time data of the alternate-runs condition were analysed separately. This was done to investigate the position-in-run effect.

The position in run had a significant response-time effect, $F(1,7)=30.1, p<$ $.01, M S E=553$. The data are shown in Figure 3. Responses on shift trials (different) were slower than those on non-shift (same) trials $(559 \mathrm{msec}$ versus $543 \mathrm{msec}$ ). With respect to the shift costs there was no significant difference between the responses to unfiltered and those to highpass-filtered stimuli.

In order to see whether the response times on non-shift trials in the alternateruns condition were different from those in the blocked condition, planned comparisons were made. They revealed that the responses in the blocked condition were significantly faster for the local level, $F(1,7)=10.1, p<.05, M S E=$ 136 , as well as for the global level, $F(1,7)=8.49, p<.05, M S E=154$. The data can be seen in Figure 3 .

With respect to the preparation time data, more time was taken to prepare on shift trials than on non-shift trials, $433 \mathrm{msec}$ versus $358 \mathrm{msec} ; F(1,7)=6.98$, $p<.05, M S E=51,501$. The data can also be seen in Figure 3.

Sequential dependencies. For the condition with the randomized target level, the data were analysed for sequential effects. It turned out that the 
responses were significantly faster on non-shift trials than on shift trials, $542 \mathrm{msec}$ versus $560 \mathrm{msec} ; F(1,7)=20.8, p<.01, M S E=253$, whereas there was no interaction with target level. There was also no significant interaction with stimulus type, i.e. the effects were not smaller for the highpass-filtered stimuli; in fact, there was even a tendency for the effects to be larger for highpass-filtered stimuli $(21 \mathrm{msec})$ than for unfiltered stimuli $(16 \mathrm{msec})$.

As can be seen in Figure 3, the response times were almost exactly the same as the corresponding data in the runs condition.

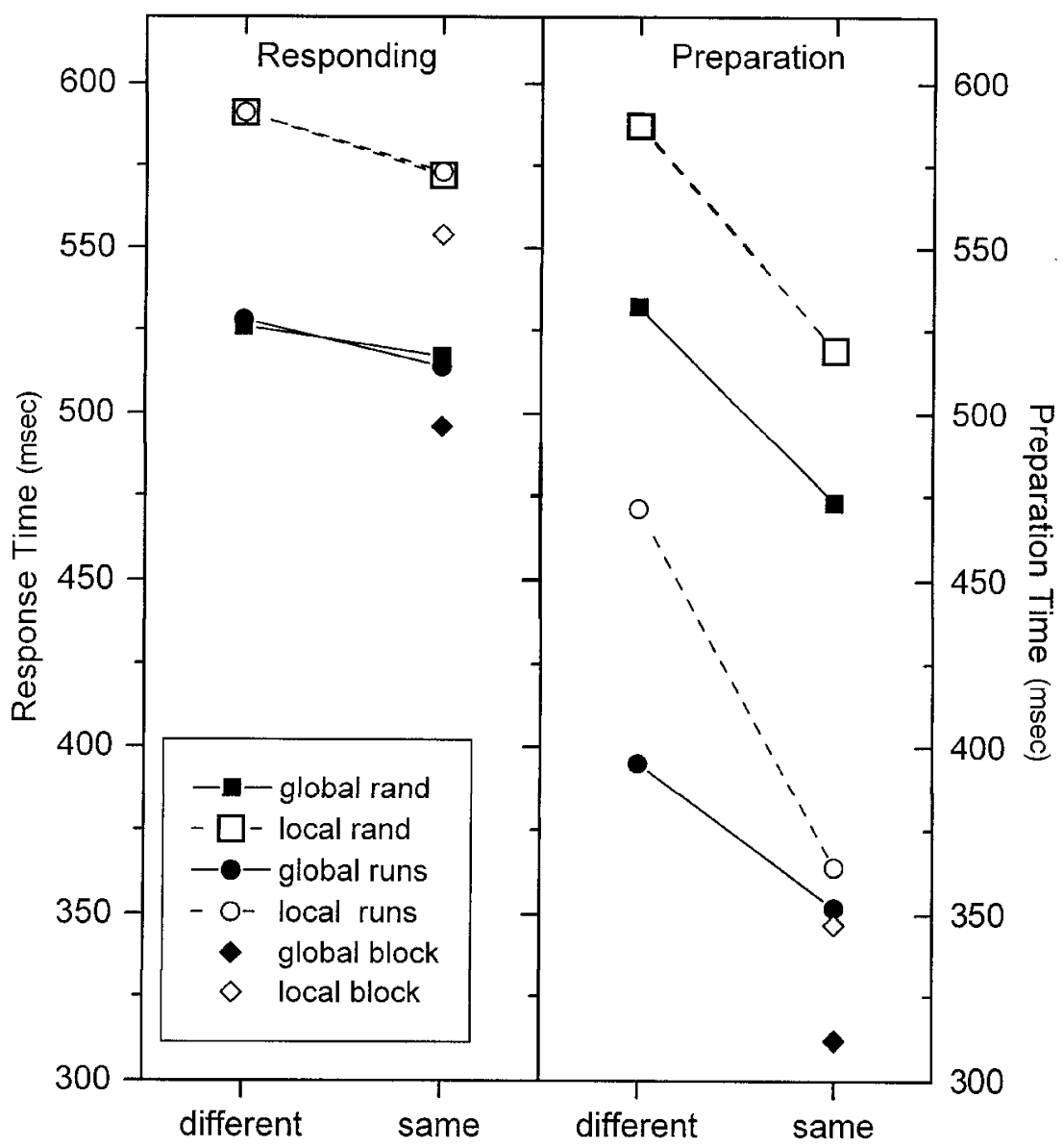

Target level in previous trial

FIG. 3. Sequential response-time and preparation-time data for the different conditions in Experiment 1. 
Also the preparation times were analysed for sequential effects. Less time was taken on non-shift trials than on shift trials, $496 \mathrm{msec}$ versus $560 \mathrm{msec} ; F(1$, $7)=5.89, p<.05, M S E=11,002$, as the data in Figure 3 show.

Error rates. Errors occurred, on average, on $6.95 \%$ of the trials. After an arcsin transformation the data were subjected to a four-factor ANOVA analogous to that of the latency data. The stimulus factor was significant, $F(1,7)=$ 9.34, $p<.05, M S E=0.0142$. More errors occurred for the highpass-filtered stimuli $(8.12 \%$ versus $5.78 \%)$. Also consistency had a significant effect, $F(1$, $7)=18.62, p<.01, M S E=0.0160$. More errors occurred for the inconsistent than for consistent stimuli $(8.77 \%$ versus $5.12 \%)$. Finally, there was a threeway interaction between stimulus type, target level, and consistency, $F(1,7)=$ $11.4, p<.05, M S E=.0026$. This interaction was due to the fact that, for the unfiltered stimuli, the consistency effect was larger for local than for global targets, whereas there was no asymmetry for the highpass-filtered stimuli. The runs data were also analysed individually, but no significant effects were found.

\section{Discussion}

One aim of Experiment 1was to investigate whether one can set up conditions under which no level-repetition effects occur. Under favourable conditions, attentional control should allow optimal preparation to select targets at the prespecified level. However, as the results reveal, this was not the case. Although the participants could prepare for as long as they wished, and even though they saw a cue indicating the size of the target pattern, there were nevertheless reliable repetition effects. These effects were not only shown by comparing performance in the constant-level condition with that in the other two, but also by regarding sequential effects within the randomized and runs conditions. What matters is the percentage of level repetitions, as predicted by the mechanismactivation hypothesis (Lamb et al., 1998). Whether the level changed deterministically or randomly had no effect; the repetition effects in the runs condition were almost identical to those in the randomized-level condition. This demonstrates that global strategies of attentional control played no role.

Concerning the spatial-frequency hypothesis, the highpass-filtered stimuli produced similar repetition effects to the unfiltered stimuli, which does not support any hypothesis, such as Robertson's (1996), which attributes the shift costs to the spatial-frequency content of the stimuli.

Although the response times were almost identical for the runs and the randomised conditions, there were differences with respect to the preparation times. More time was taken for preparation in the condition with randomized levels than for preparation in the alternate-runs condition. Although this difference might, at least partly, be due to the fact that the participants had always to 
encode the cue in the randomised but not in the runs condition, it could also reflect some differences in effort for preparation. This interpretation is supported by the result that the preparation times were shorter in the constant target-level condition than in the runs condition. Moreover, more preparation time was taken for processing the local level than for processing the global one, which was even the case in conditions with a constant target level.

\section{EXPERIMENT 2}

Since the effects of level repetition on response time were rather small, compared with those on the preparation times, the question arises as to what extent participants have control of the differential allocation of resources for preparation and performance. In other words, if participants would have only a restricted time interval for preparation, would the level-repetition effect on response time increase? This question was investigated in the next experiment, in which the target level was always randomized, and where also a condition with an experimenter-paced trial rate was included. If the participants use the preparation time for allocating their attention properly, then larger responsetime costs should occur without such an interval. However, participants might also use the preparation time for resting. To investigate this, a further condition was included in which the trial rate was self paced, but where no cue was available during this period. Finally, in a third condition the trial rate was self paced, and a cue was present during the preparation interval, as in Experiment 1.

In addition to the above manipulation, the interval between the cue and the stimulus (ISI) was varied to gain more detailed information on the time course of the top-down processes involved. The stimulus was presented either $100 \mathrm{msec}$ or $400 \mathrm{msec}$ after the cue had disappeared, and shorter response times were expected for the longer ISI, but only for the first two preparation conditions.

\section{Method}

The same apparatus and a similar method as in the first experiment was applied. Eight people (two male and six female), who ranged in age between 20 and 38 years, participated. All had normal or corrected-to-normal visual acuity. The stimuli were the unfiltered compound letters from Experiment 1. Letters were presented randomly either left or right of the fixation cross (with $0.95^{\circ}$ eccentricity). The target level was always randomized across trials.

There were three different preparation conditions (see Figure 4). One was similar to the condition with the randomized target level in Experiment 1. After the cue had arrived, participants could start the trial by themselves without time pressure. Then, either $100 \mathrm{msec}$ or $400 \mathrm{msec}$ after the cue had disappeared, the 


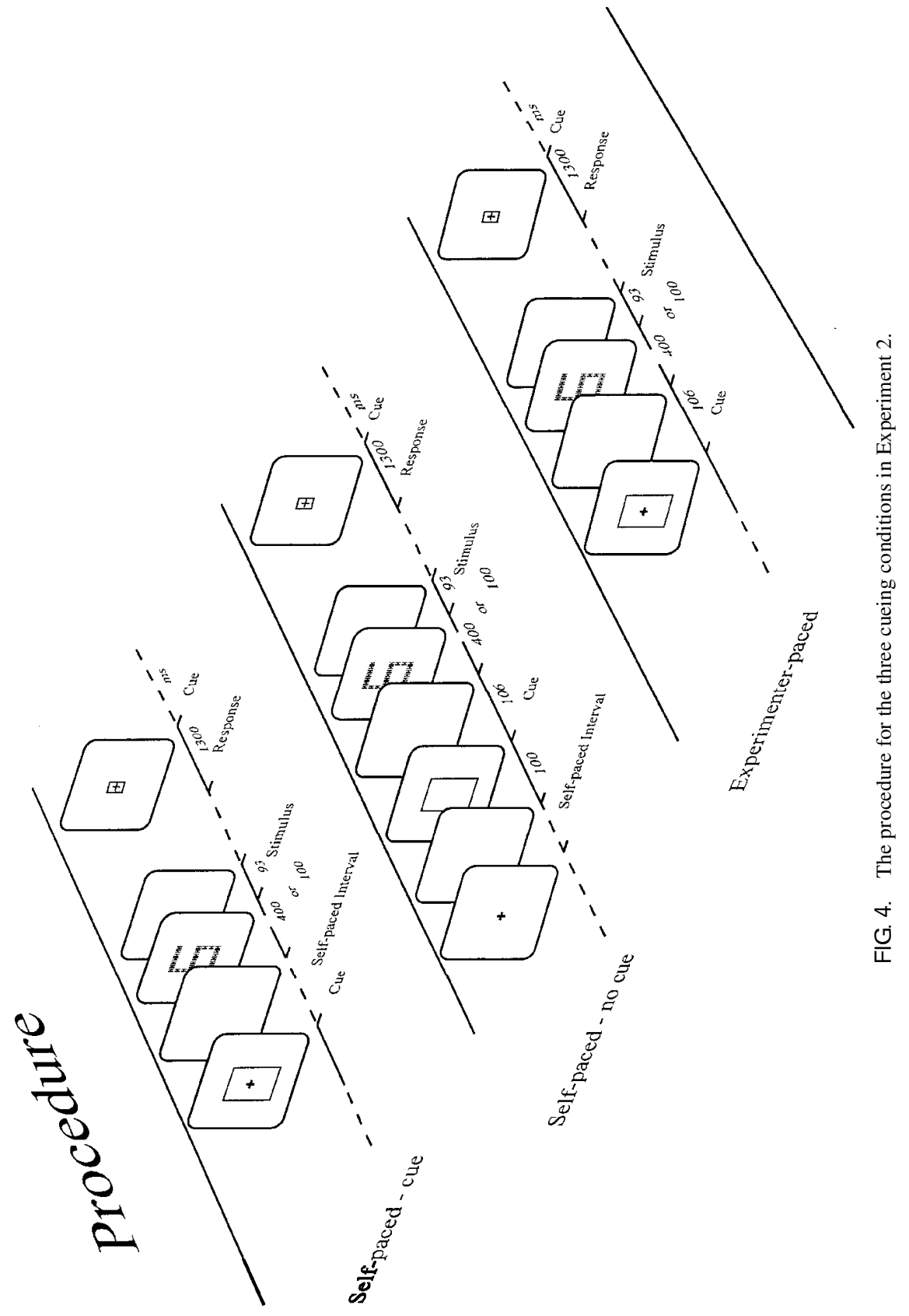


stimulus was presented. Finally, 1300msec after the response, the next cue appeared.

In a second condition the trial rate was also self paced. However, the participants saw only the fixation cross in the preparation interval. Then, $100 \mathrm{msec}$ after they had started the trial, the cue appeared for $106 \mathrm{msec}$, and either $100 \mathrm{msec}$ or $400 \mathrm{msec}$ thereafter the stimulus was presented.

Finally, in a third condition the participants could not start the trials by themselves. In this case, $1300 \mathrm{msec}$ after the last response the cue appeared together with the fixation cross for $106 \mathrm{msec}$, and then, either $100 \mathrm{msec}$ or $400 \mathrm{msec}$ later, the stimulus was presented.

Thus, four experimental factors were varied: preparation condition (no preparation, preparation without cue, preparation with cue), target level (local, global), consistency (consistent, inconsistent), and ISI (100, 400). Altogether there were 24 different conditions, where the preparation conditions were blocked. Each block was run six times, where the first run was considered as training. The order of blocks was counterbalanced across the participants. Altogether there were 80 experimental trials for each condition and participant.

\section{Results}

Response times. The mean latencies were subjected to a four-factor ANOVA with repeated measures on all factors: preparation condition (no preparation, preparation without cue, preparation with cue), ISI (100, 400), target level (global, local), and consistency (consistent, inconsistent).

The preparation conditions differed significantly, $F(2,14)=15.795, p<$ $.001, M S E=7351$. Bonferroni t-tests (for paired comparisons) computed across conditions and participants revealed that responses in the no-preparation condition were significantly slower than those in the preparation condition without cue, $656 \mathrm{msec}$ versus $613 \mathrm{msec} ; t(63)=9.40, p<.001$, and that the responses in the latter condition were slower than those in the preparation condition with cue, $598 \mathrm{msec} ; t(63)=4.15, p<.001$.

Also the ISI factor had a significant main effect, $F(1,7)=79.4, p<.001$, $M S E=2230$. Responses after the longer ISI were faster $(601 \mathrm{msec})$ than those after the shorter ISI $(644 \mathrm{msec})$. Finally, both the target-level factor [global: 579msec, local: 667msec; $F(1,7)=55.8, p<.001, M S E=13,051]$, and the consistency factor were significant [consistent: $609 \mathrm{msec}$, inconsistent: 636msec; $F(1,7)=41.0, p<.001, M S E=1660]$.

Of the interactions only that between target level and consistency was reliable, $F(1,7)=12.6, p<.01, M S E=1,784$. Consistency had larger effects for local targets $(645 \mathrm{msec}$ versus $687 \mathrm{msec})$ than for global targets $(573 \mathrm{msec}$ versus $585 \mathrm{msec}$ ). Unexpectedly, there was no significant interaction between preparation condition and ISI (ISI effect: $45,47,47 \mathrm{msec}$ ). The data can be seen in Figure 5. 




FIG. 5. Mean response-time data for the different conditions in Experiment 2.

Preparation times. A comparison between the preparation times for the two self-paced conditions revealed a significant difference, $F(1,7)=5.86, p<$ $.05, M S E=3486$. More time was taken when a cue was available (576msec) relative to when there was no cue $(526 \mathrm{msec})$.

Errors. The mean error rate was 7.68\%. Only the consistency factor was significant [consistent: $5.01 \%$, inconsistent: $10.4 \% ; F(1,7)=45.3, p<.001$, $M S E=0.0296]$. 
Sequential dependencies. All response-time data were analysed for levelrepetition effects with preparation condition as additional factor. Level repetition had a significant effect, $F(1,7)=5.92, p<.05, M S E=2582(630 \mathrm{msec}$ versus $655 \mathrm{msec}$ ). However, there was no interaction with the preparation conditions.

\section{Discussion}

The results show, as expected, that the experimenter-paced condition produced the slowest responses. In this condition the participants had a restricted time interval for cue encoding and preparation. When the preparation-time interval was controlled by the participants, then the response times decreased. If additionally a cue was available during the self-paced preparation interval, then the response times decreased again. This latter effect could be accounted for by the additional time used for encoding the cue; alternatively, one could speculate that it reflects voluntary attentional control. However, the latter interpretation can be questioned, because the level-repetition effects were unaffected by the different preparation conditions.

That the effect of voluntary control was quite restricted is also shown by the ISI effects. As expected, performance improved with an increasing ISI; however, ISI did not interact with the preparation conditions. One would have expected the short ISI to have disrupted performance more in the experimenterpaced condition than in the condition where the participants had enough time for preparation, and where they saw the cue during the interval. That this result did not occur indicates that the ISI effect does not reflect time limitations with respect to the preparation of the processing of specific stimulus features; rather, the effect seems to reflect non-specific attentional mechanisms.

The preparation times were generally rather small compared with those in the first experiment. This suggests that the experience gained by the participants in the experimenter-paced condition, namely that attention shifting can be achieved without extensive preparation, led them to change their strategy and to use less preparation time in the self-paced conditions as well.

Although it remains open what exactly the participants did in the different intervals in the different conditions, the data clearly show that level-repetition effects are largely independent of different cueing conditions and of the ISI.

\section{GENERAL DISCUSSION}

The present experiments investigated the factors determining the efficiency of target-level selection (and/or processing) in a global/local identification task. If we consider various results (e.g. Hübner, 1997, 1998; Lamb \& Yund, 1996b; Ward, 1982), it turns out that there are at least three mechanisms that could affect efficiency of selection in such tasks. First, attentional resources could be 
allocated by voluntary control to a given target level. That such a control is possible, at least up to a certain degree, has been demonstrated by negative effects of invalid cues, i.e. by the fact that cues directing attention to the non-target level produce response-time costs (e.g. Robertson et al., 1993). On the other hand, even when valid cues are given to select local targets, a global responsetime advantage cannot generally be prevented. This can be interpreted either as the result of sensory effects, or as the result of a stronger saliency of the global level, which, therefore, attracts attention automatically (i.e. some form of exogenous control of attention). Finally, as we have demonstrated in the present experiments, the availability of valid cues, even in combination with a selfpaced procedure, does not prevent level-repetition effects. These effects demonstrate that there are proactive exogenous factors, which affect subsequent selection efficiency, and which resist voluntary control.

Although both the global response-time advantage and the interference asymmetry were large in the present experiments, the level-repetition effects were nevertheless symmetric, i.e. the repetition effects were similar for responses to the global and to the local level, which is in line with other results (e.g. Lamb et al., 1998; Robertson, 1996; Ward, 1982). This symmetry, despite the large response-time differences between global and local, suggests that the level-repetition effect occurs at a relatively abstract stage of processing. However, no evidence was found for the hypothesis, stated by Robertson (1996), that repetition effects are related to the spatial-frequency content of the stimuli. In Experiment 1, where highpass-filtered patterns were employed, the repetition effects were similar for filtered and unfiltered stimuli. Therefore, the levelrepetition effects must be due to mechanisms other than the weighting or preactivation of specific spatial-frequency channels (see also Lamb \& Yund, 1996a, b; Lamb et al., 1999).

In a more general approach, Lamb et al. (1998) proposed that the processing of a given target level has an advantage when the appropriate level-specific mechanisms are still active from the processing of the same level on the preceding trial. From this "mechanism activation" hypothesis they predicted that the percentage of level repetition is a crucial factor for repetition effects. This was confirmed in the present study. Compared with a condition in which $100 \%$ repetitions (constant-level) occurred, those with 50\% repetitions produced reduced performance, and it did not matter whether the target level was randomized or followed a deterministic pattern of runs. The benefit in performance, on repeat trials, was almost identical in both cases. Also the interference effects between the levels were increased in the two variable-level conditions. Thus, one could speculate that repetition benefits might be due to reduced interference. However, the fact that the repetition effects were symmetric, despite a large asymmetric interference between the levels, does not support such an interpretation. Also, highpass filtering reduced the interference effects, but did not affect the repetition effects. 
Although the response-time data did not differ between the randomizedlevel and the runs condition, the preparation-time data did. Participants took longer to prepare in the randomized condition. There were also different preparation times for global and local levels. More time was spent by the participants to prepare processing of the local level than to prepare processing of the global one; this difference occurred even for the constant conditions. The latter result suggests that the processing of local features is harder than that of global features, and that the participants tried to prepare themselves accordingly. This could indicate that the preparation-time differences for the different conditions in Experiment 1 also reflect different amounts of effort for preparation. In the randomized-level condition the participants presumably tried harder to prepare themselves for the processing of the required level, although it had no effect. Such as interpretation is in line with results obtained by Strayer and Kramer (1994), whose participants reported that they were able to use cues for altering their performance, though, objectively, this was not the case.

In Experiment 2 here, participants had in one condition no control of the duration of the preparation interval. Response times then increased. This is perhaps not surprising, and can be the result of various processes, such as the lack of resting time, restricted time for cue encoding, etc. However, and this is most important for the present objective, even in this case the repetition effects remained unaffected. Taken together, the results of the present experiments demonstrate that there are residual level-repetition effects, which are unaffected by voluntary control and even by the interval length between the trials. Thus, they seem to originate from automatic and stimulus driven processes.

Interestingly, similar residual effects have also been observed in experiments where, instead of shifting attention between stimulus dimensions, shifting attention between mental tasks was required (e.g. Allport, Styles, \& Hsieh, 1994; Rogers \& Monsell, 1995). These experiments have shown that the reconfiguration of the mental system for performing a certain task cannot be attained completely by top-down control. For instance, in one of Rogers and Monsell's (1995) experiments only about one-third of the shift costs could be eliminated by preparation. How could these residual shift costs be explained? Allport et al. (1994) assumed that the costs are due to proactive interference from the recently performed task. They hypothesized that response-relevant stimulus features are linked via a stimulus-response association with the respective responses. Therefore, a stimulus automatically triggers a tendency to respond even if a different task is required, which leads to interference between the tasks. The independence of the shift costs from ITI led them to conclude that the proactive interference from the old task dissipates rather slowly and gradually across several trials, an aspect that they called "task-set inertia". Thus, according to this proposal, a mental shift not only requires the preparation of the new task but also the disengagement from the last task, which is thought not to be possible by endogenous control alone. 
Obviously, Allport et al.'s approach is rather general and accounts not only for task-set shifts, but can also be applied to shifts between stimulus dimensions. Indeed, Lamb et al.'s (1998) “mechanism activation” hypothesis is rather similar to the task-set inertia hypothesis. However, the fact that the asymmetric within-trail interference between the levels does not affect level-repetition effects, suggests that the proactive task interference occurs at a different stage of processing, where the task of identifying letters at a global level has the same status as that of identifying letters at a local one. Thus, the categorical stage of level processing, as proposed by Robertson et al. (1993), can more generally be thought of in terms of task set. Task-set inertia seems to produce characteristic repetition effects, which are similar for various tasks.

To sum up, the results of the present experiments demonstrate that levelrepetition effects are widely unaffected by voluntary control mechanisms, by the spatial-frequency content of the stimuli, by the interval between the trials, by the interval between cues and stimulus, by the arrangement of level repetitions, and by the interference between the levels. They seem to be automatic and related to task-set shift effects.

\section{REERENCES}

Allport, A., Styles, E.A., \& Hsieh, S. (1994). Shifting intentional set: Exploring the dynamic control of tasks. In C. Umiltà \& M. Moscovitch (Eds), Attention and performance XV (pp. 421-452). Cambridge, MA: MIT Press.

Egeth, H.E., \& Yantis, S. (1997). Visual attention: Control, representation, and time course. Annual Review of Psychology, 48, 269-297.

Hoffman, J.E. (1980). Interaction between global and local levels of a form. Journal of Experimental Psychology: Human Perception and Performance, 6, 222-234.

Hübner, R. (1997). The effect of spatial frequency on global precedence and hemispheric difference. Perception and Psychophysics, 59, 187-201.

Hübner, R. (1998). Hemispheric differences in local-global processing revealed by same-different judgements. Visual Cognition, 5, 457-478.

Hughes, H.C., Nozawa, G., \& Kitterle, F. (1996). Global precedence, spatial frequency channels, and the statistics of natural images. Journal of Cognitive Neuroscience, 8, 197-230.

Johnston, W.A., \& Dark, V.J. (1986). Selective attention. Annual Review of Psychology, 37, 4375.

Kinchla, R.A. (1992). Attention. Annual Review of Psychology, 43, 711-742.

Kinchla, R.A., Solis-Macias, V., \& Hoffman, J. (1983). Attending to different levels of structure in a visual image. Perception and Psychophysics, 33, 1-10.

Lamb, M.R., London, B., Pond, H.M., \& Whitt, K.A. (1998). Automatic and controlled processes in the analysis of hierarchical structure. Psychological Science, 9, 14-19.

Lamb, M.R., \& Robertson, L.C. (1988). The processing of hierarchical stimuli: Effects of retinal locus, location uncertainty, and stimulus identity. Perception and Psychophysics, 44, $172-$ 181.

Lamb, M.R., \& Yund, E.W. (1996a). Spatial frequency and interference between goals and local levels of structure. Visual Cognition, 3, 193-219. 
Lamb, M.R., \& Yund, E.W. (1996b). Spatial frequency and attention: Effects of level, target, and location repetition on the processing of global and local forms. Perception and Psychophysics, 58, 363-373.

Lamb, M.R., Yund, E.W., \& Pond, H.M. (1999). Is attentional selection to different levels of hierarchical structure based on spatial frequency? Journal of Experimental Psychology: General, 128, 88-94.

Miller, J. (1981). Global precedence in attention and decision. Journal of Experimental Psychology: Human Perception and Performance, 7, 1161-1174.

Navon, D. (1977). Forest before trees: The precedence of global features in visual perception. Cognitive Psychology, 9, 353-383.

Posner, M.I. (1980). Orienting of attention. Quarterly Journal of Experimental Psychology, 32, $3-25$.

Robertson, L.C. (1996). Attentional persistence for features of hierarchical patterns. Journal of Experimental Psychology: General, 125, 227-249.

Robertson, L.C., Egly, R., Lamb, M.R., \& Kerth, L. (1993). Spatial attention and cueing to global and local levels of hierarchical structure. Journal of Experimental Psychology: Human Perception and Performance, 19, 471-487.

Rogers, R.D., \& Monsell, S. (1995). Costs of a predictable switch between simple cognitive tasks. Journal of Experimental Psychology: General, 124, 207-231.

Shiffrin, R.M. (1988). Attention. In R.C. Atkinson, R.J. Hernstein, G. Lindzey, \& R.D. Luce (Ed), Stevens handbook of experimental psychology, Vol. 2. (pp. 739-811). New York: Wiley.

Strayer, D.L., \& Kramer, A.F. (1994). Strategies and automaticity: II. Dynamic aspects of strategy adjustment. Journal of Experimental Psychology: Learning, Memory, and Cognition, 20, 342-365.

Ward, L.M. (1982). Determinants of attention to local and global features of visual forms. Journal of Experimental Psychology: Human Perception and Performance, 8, 562-581. 